\title{
换位思考联合共情护理对急性冠脉综合征患者心理状态及 预后的影响
}

\author{
Effects of Transposition Thinking and Empathy Nursing on Psychological Status and Prognosis of \\ Patients with Acute Coronary Syndrome \\ 薛亚亚 \\ Yaya Xue
}

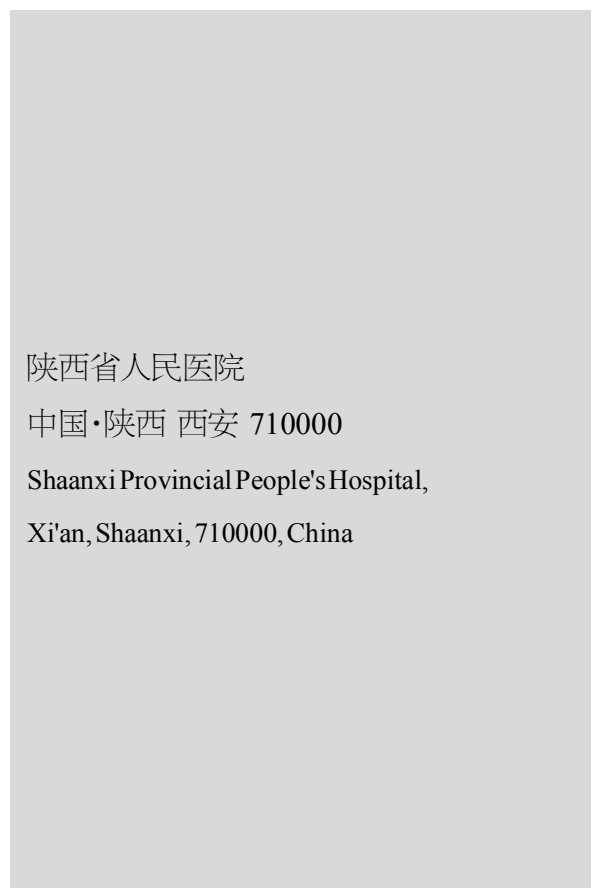

\section{1 资料与方法}

\section{1 一般资料}

本研究采取随机的方式将我院 2018 年 1 月至 2019 年 6 月期间治疗的 126 例急性冠脉综合征患者分为观察组 $(63$ 例)、对照组 (63 例)。观察组与对照组分别有 31 例、32 例男性 患者, 32 例、31 例女性患者; 患者平均年龄分别为（62.21土 $2.68)$ 岁、(61.53 3.88$)$ 岁。P>0.05, 基础数据差异不具有统计学 意义。

\section{2 方法}

1.2 .1 对照组患者护理方法

医护人员组织对照组患者开展健康讲座, 进行群体心理 护理等常规护理。

1.2 .2 观察组患者护理方法

(1)创建护理小组: 科室对组内医护人员进行人文理论、共 情知识、护理认知、沟通方式等培训; (2)制定护理计划: 护理
前, 医护人员利用焦虑自评量表及抑郁自评量表检测观察组 患者的心理状态, 以检测结果为依据为其制定针对性护理计 划; (3)加强沟通交流: 医护人员应采取鼓励性、积极、阳光的语 言与患者进行沟通交流, 如发现患者的心理状态不佳, 应立即 对其信息进行汇总，反馈后给予其有针对性的心理疏导; (4)心 理护理: 医护人员应通过心理教育、情绪疗法、放松训练等方 式给予患者心理护理, 通过科学合理的干预使其树立治疗信 心, 战胜病魔, 早日康复; (5)护理评价: 医护人员应对心理护理 结果进行正确评析, 及时找到护理中存在的不足, 并以此为基 础更改护理方案, 从而加强护理效果 ${ }^{[1]}$ 。

\section{3 观察指标}

护理结束后，医护人员将观察组与对照组患者的 SAS、 SDS 评分及预后情况进行分析对比。

\section{4 统计学方法}

本研究使用 SPSS22.0 系统进行统计分析, 正态分布计量资 料以 $\bar{x} \pm \mathrm{s}$ 表示, 采用 $t$ 检验, 等级资料采用秩和检验, 计数资料 
临床医学研究 Clinical Medicine Research

以百分比表示, 采用 $\chi^{2}$ 检验, 以 $P<0.05$ 为差异有统计学意义。

\section{2 结果}

\section{1 观察组与对照组患者护理前后 SAS、SDS 评分对比}

观察组与对照组患者护理前后 SAS、SDS 评分具体如表 1 所示:

表 1 观察组与对照组患者护理前后 SAS、SDS 评分对比

\begin{tabular}{|c|c|c|c|c|}
\hline \multirow{2}{*}{ 组别 } & \multicolumn{2}{|c|}{ SAS } & \multicolumn{2}{|c|}{ SDS } \\
\hline & 护理前 & 护理后 & 护理前 & 护理后 \\
\hline 观察组 & $37.56 \pm 8.22$ & $22.53 \pm 4.95$ & $37.64 \pm 7.86$ & $20.02 \pm 3.52$ \\
\hline 对照组 & $37.11 \pm 7.95$ & $29.69 \pm 5.68$ & $38.96 \pm 5.24$ & $28.54 \pm 5.18$ \\
\hline$P$ & 0.512 & 5.698 & 0.538 & 6.253 \\
\hline$t$ & 0.623 & 0.001 & 0.436 & 0.002 \\
\hline
\end{tabular}

\section{2 观察组与对照组患者预后情况对比}

观察组与对照组患者预后情况具体如表 2 所示:

表 2 观察组与对照组患者预后情况对比

\begin{tabular}{c|c|c|c|c}
\multicolumn{5}{c}{} \\
\hline 组别 & 心肌梗死 & 肝功能异常 & 血管重建 & 胃肠道反应 \\
\hline 观察组 & 0 & 1 & 1 & 2 \\
\hline 对照组 & 4 & 4 & 3 & 5 \\
\hline$\chi^{2}$ & 3.246 & 5.616 & 5.133 & 5.663 \\
\hline$P$ & 0.000 & 0.002 & 0.001 & 0.026 \\
\hline
\end{tabular}

\section{( 上接第 26 页)}

分钟。同时, 以 TDP 理疗患者的下腹部, 时间也为 30 分钟, 并 保持 $0.3 \sim 0.4 \mathrm{~m}$ 的距离。最后, 在针刺、TDP 理疗结束后, 在患者 肾俞、第十七椎穴、八髎位置进行拔罐治疗, 时间为 10 15 分 钟。每日治疗一次,一月治疗十次,每两次间隔一日,可于经期 后进行, 避免因经期中断。十次为一疗程, 连续治疗三疗程, 三 疗程后, 观察治疗效果。

\section{3 评判标准}

对比两组患者的治疗效果。治疗效果分显著、有效、一般 三种标准。治疗效果显著, 表现为治疗结束后患者的身体完全 康复, 不适症状尽数消失或医疗检测中患者的病灶部位恢复 正常; 治疗效果有效, 表现为治疗结束后患者身体的不适症状 好转显著或医疗检测中患者的病灶部位状况明显改善; 治疗 效果一般, 表现为治疗结束后患者的身体状况无明显变化或 医疗检测中患者的病灶部位无明显变化。另外, 治疗总有效 率=显著+有效。

\section{4 统计学处理}

本研究数据均使用 SPSS22.0 系统进行处理, 计量资料以 均数 \pm 标准差 $(\bar{x} \pm \mathrm{s})$ 表示, 采用 $t$ 检验, 计数资料以百分比表 示, 采用 $\chi^{2}$ 检验, $P<0.05$ 为差异有统计学意义。

\section{3 讨论}

医疗技术水平的提升降低了急性冠脉综合征的死亡概 率,但患者的病情愈后情况有待加强。该病愈后对患者的生活 质量有了全新的要求，因此在急性冠脉综合征患者的护理过 程中, 医护人员应对其进行有针对性的心理疏导。共情护理能 够有效提升急性冠脉综合征患者的护理有效率。根据此次分 组研究结果可知, 护理后, 观察组患者的 SAS、SDS 评分显著 优于对照组患者, $P<0.05$; 观察组患者心肌梗死、肝功能异常、 血管重建、胃肠道反应的情况均优于对照组患者, $P<0.05$ 。

\section{4 结语}

综上所述，医护人员给予急性冠脉综合征患者换位思考 联合共情护理, 对其心理状态及病情预后有十分积极的影响, 而且能够改善患者的生活质量, 对病情的恢复有促进作用, 该 护理方式的护理疗效极为显著,应推广使用。

\section{参考文献}

[1]冒云霞,李英,黄荔荔.换位思考联合共情护理对急性冠脉综合 征患者心理状态及预后的影响[J].齐鲁护理杂志,2019,25(19):82-84.

[2]朱萍,杨承健.双心护理联合放松训练对急性冠脉综合征患者 心理状态的影响[J].护理学杂志,2017,32(21):89-90+96.

\section{2 结果}

观察组的治疗总有效率显著高于对照组 $(P<0.05)$, 详情 见表 1 。

表 1 两组患者治疗效果对比

\begin{tabular}{c|c|c|c|c}
\hline 组别 & 显著/例 & 有效/例 & 一般/例 & 总有效率/\% \\
\hline 对照组 & 10 & 24 & 16 & 68.0 \\
\hline 观察组 & 20 & 24 & 6 & 88.0 \\
\hline$\chi^{2}$ & 4.762 & 0.000 & 5.828 & 5.828 \\
\hline$P$ & 0.029 & 1 & 0.016 & 0.016 \\
\hline
\end{tabular}

\section{3 结语}

中医学认为, 女性乃热盛阳六之体, 在外来因素的影响 下, 气血容易亏虚, 湿热毒邪乘机侵入体内, 与人体血互结然 后下注, 从而损伤人体带脉, 进而引发慢性盆腔炎。

在本研究中，观察组患者的治疗总有效率显著高于对照 组 $(P<0.05)$ 。由此表明, 在慢性盆腔炎患者治疗中采取针刺与 TDP 理疗结合拔罐治疗可以起到良好的治疗效果, 促进患者 的身体康复。

\section{参考文献}

[1]李艺. 慢性盆腔炎妇产科临床治疗效果观察[J].当代临床医刊, 2016,29(5):2572. 